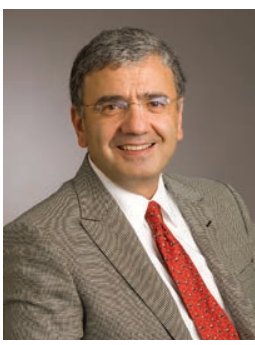

\title{
CARDIOVASCULAR IMAGING IN THE 21ST CENTURY
}

\author{
William A. Zoghbi, M.D.
}

Houston Methodist DeBakey Heart \& Vascular Center, Houston Methodist Hospital, Houston, Texas

W.A. Zoghbi, M.D.

The past 50 years have seen amazing developments in cardiovascular imaging, with resultant impact on medical diagnosis, patient management, and outcome. From a simple electrocardiogram and stethoscope at the bedside, diagnostics have evolved within a relatively short period to sophisticated imaging-showing details of various cardiac structures and unraveling cardiac physiology and pathophysiology. When addressing this topic, I am always reminded of a patient of mine with rheumatic valvular disease who had three mitral valvular repairs, the first being in the early 1960s. She kept a true carbon copy of her initial medical evaluation, which included a history and detailed physical examination of her mitral stenosis, complete with a timing of the opening snap, a chest X-ray, and a barium swallow evaluating her left atrial enlargement. This was the extent of her noninvasive evaluation.

Fast forward to the present, where imaging modalities can capture not only the valve leaflets in real-time 3-dimensions but also assess the size and function of the cardiac chambers, beat-to-beat gradient across the valve, and pulmonary artery pressure, all noninvasively. Imaging in the 21st century touches all areas of cardiovascular medicine-ventricular function, coronary artery disease, valvular, congenital and vascular disease, and, more recently, guiding cardiac interventions-making it an essential component of training and practice of cardiovascular professionals. In this issue of the Methodist DeBakey Cardiovascular Journal, I have asked leading experts in cardiovascular imaging from the Houston Methodist DeBakey Heart \& Vascular Center and Texas Medical Center to discuss innovations in each of the noninvasive imaging modalities, including echocardiography, nuclear imaging, cardiac computed tomography (CT), and cardiac magnetic resonance (CMR). The authors have nicely detailed the latest advances in technology as well as current and potential clinical applications in the foreseeable future.

With the advent of new imaging technologies for evaluating and diagnosing cardiovascular disorders and the evolution of catheterbased techniques for treating structural heart disease, so too has emerged a new field of interventional imaging. The imager is currently an integral member of the heart team, involved in both shared decision making and the actual procedure for real-time guidance and assessment of immediate results. The breadth and importance of interventional imaging and the role of the imager is also discussed in this issue.

From echocardiography and nuclear to CT and CMR, imaging technologies have increased in number and complexity, with some overlapping applications. Physicians in training programs or already in practice must have enough familiarity with the various imaging modalities to order the most appropriate test that best addresses the clinical question. To this end, appropriate-use criteria for imaging have been published by the American College of Cardiology and are discussed in this issue. Admittedly, more than one imaging modality may be necessary to address a physiologic and anatomic issue. However, avoiding unnecessary testing is critical in a health care system that nowadays is driven more by value, efficiency, patient convenience, and safety, particularly when imaging involves radiation. Since we are stewards of such a system, future research and development should focus on determining the best methodology to diagnose and manage patients in a multimodality imaging world, especially one in which new digital gadgets and handheld devices can provide early diagnostics at the bedside.

Selecting the most effective test is contingent on obtaining a thorough history and physical examination, without which one may be at a loss of what is needed and may inadvertently order excessive testing. A recent case illustrates this point. An older prominent scientist had three syncopal episodes and was given a diagnosis of arrhythmic syncope; surprisingly, he had not been examined, prompting his selfreferral to our institution. A physical examination revealed a murmur consistent with severe aortic stenosis, which was corroborated by an echocardiogram. One can have all imaging modalities available, but if not directed to answer a particular clinical question based on a detailed history and proper examination, one may err in many different directions.

The future of cardiovascular imaging is bright, the field so important, that one may regard imaging as an essential competence, not necessarily expertise, of every cardiologist. In a subsequent article, I offer a glimpse of the future of cardiovascular imaging and identify potential areas of research and remaining challenges.

I want to thank the authors for contributing thoughtful, comprehensive, and visionary articles on cardiovascular imaging and Dr. William Winters for the opportunity to be the guest editor of this issue. 